\title{
Sensors Management in Robotic Neurosurgery: the ROBOCAST project
}

\author{
Alberto Vaccarella, Mirko Daniele Comparetti, Andinet Enquobahrie, Giancarlo Ferrigno and Elena \\ De Momi
}

\begin{abstract}
Robot and computer-aided surgery platforms bring a variety of sensors into the operating room. These sensors generate information to be synchronized and merged for improving the accuracy and the safety of the surgical procedure for both patients and operators. In this paper, we present our work on the development of a sensor management architecture that is used is to gather and fuse data from localization systems, such as optical and electromagnetic trackers and ultrasound imaging devices. The architecture follows a modular client-server approach and was implemented within the EU-funded project ROBOCAST (FP7 ICT 215190). Furthermore it is based on very well-maintained open-source libraries such as OpenCV and Image-Guided Surgery Toolkit (IGSTK), which are supported from a worldwide community of developers and allow a significant reduction of software costs.

We conducted experiments to evaluate the performance of the sensor manager module. We computed the response time needed for a client to receive tracking data or video images, and the time lag between synchronous acquisition with an optical tracker and ultrasound machine.

Results showed a median delay of 1.9 ms for a client request of tracking data and about $\mathbf{4 0}$ ms for US images; these values are compatible with the data generation rate $(20-30 \mathrm{~Hz}$ for tracking system and 25 fps for PAL video).

Simultaneous acquisitions have been performed with an optical tracking system and US imaging device: data was aligned according to the timestamp associated with each sample and the delay was estimated with a cross-correlation study. A median value of $230 \mathrm{~ms}$ delay was calculated showing that realtime 3D reconstruction is not feasible (an offline temporal calibration is needed), although a slow exploration is possible. In conclusion, as far as asleep patient neurosurgery is concerned, the proposed setup is indeed useful for registration error correction because the brain shift occurs with a time constant of few tens of minutes.
\end{abstract}

\section{INTRODUCTION}

$\mathrm{T}$ Echnologically-advanced surgical platforms exploit navigation and/or robotic systems to improve the outcome of procedures in several fields, such as orthopedic surgery [1], abdominal surgery [2], and neurosurgery [3].

This work was supported by the EU Project Grant ROBOCAST FP7ICT-215190 and Scuola Interpolitecnica di Dottorato, Politecnico di Milano, Bari e Torino.

A. Vaccarella is with Politecnico di Milano, Bioengineering Department, Neuroengineering and Medical Robotics Laboratory, Piazza Leonardo da Vinci 32, 20133 Milano, Italy. (e-mail: alberto.vaccarella@mail.polimi.it)

M.D. Comparetti, G. Ferrigno and E. De Momi are with Politecnico di Milano, Bioengineering Department, Neuroengineering and Medical Robotics Laboratory, Milano, Italy.

A. Enquobahrie is with Kitware Inc., Carrboro, NC, USA
Thus, the need for different sensors in the operating room (OR) has highly increased in the past two decades with the purpose of improving the accuracy and the safety of surgical procedures for both patients and operators. The main typologies of sensors used in the OR include localization systems, inertial sensors, force sensors, and intra-operative imaging devices.

Surgical navigation systems usually rely on information acquired using localization systems (mechanical, optical, or electromagnetic) in order to localize the pose of surgical instruments and to overlay them on the CT or MR images to assist surgeons during procedures [4].

Inertial Measurement Unit (IMU) allows for determination of an object's position and orientation at a high rate, even with no line of sight; on the other hand, IMUs are limited by noise amplification and drift, due to the double integration of the acceleration signal. They have recently been proposed in robotic surgery, in addition to an optical tracking system, to overcome the limitation of localization systems. In [5] Kalman-based sensor data, fusion has been proposed for a robot-controlled algorithm for motion compensation of the patient.

The use of force sensors has been recently investigated in order to provide haptic feedback to tele-operated surgical robots; surgical tools have been endowed with strain gauges [6], [7] or optical, fiber-based force sensors in order to measure small interaction forces in the range of $2.5 \mathrm{~N}$ with a resolution of $0.04 \mathrm{~N}$ [8].

Intra-operative imaging devices, which include Magnetic Resonance Imaging (MRI), Computed Tomography (CT), Fluoroscopy, and Ultrasonography (US), are used to update preoperative images and thus improve the accuracy of navigation systems; this is of the utmost importance when dealing with soft tissues. In neurosurgery, even a small aperture in the skull (mini-invasive procedure also known as keyhole neurosurgery) results in an intra-operative brain deformation that is known as brain shift (mainly due to cerebrospinal-fluid drainage). Different approaches have been proposed to estimate brain shift based on intraoperative MRI [9], 2D ultrasound images [10], and 3D ultrasound reconstruction (Freehand 3D US) [11]. The use of ultrasound imaging devices is of great interest because of their relatively low cost, small encumbrance in the OR, low invasiveness, and high frame rate. In particular, Freehand 3D US is achieved by tracking the pose in space of an ultrasound probe, which was previously calibrated with 
respect to the localization system. Temporal alignment of tracking data and US images is a demanding requirement [12] to ensure accuracy of the reconstruction; this is achieved by estimating the overall latency between images and tracking data through a temporal calibration procedure.

Sensor information is crucial, especially when the surgical intervention is assisted by robots. Robotic systems have been used in surgery for tool positioning, particularly for orthopedic or neurosurgical procedures. The robot is traditionally directly calibrated with respect to the patient prior to the procedure, so that the relative positions are kept steady [13]. The use of localization systems allows the detection of any possible displacement.

The ROBOCAST project (FP7-ICT-2007-215190) proposes a hybrid, navigated, robotic system for neurosurgery, where an integrated use of multimodal tracking systems, force sensors, and an ultrasound imaging device would improve the safety of the procedure by providing redundant information for the robot control loop and for the surgeons [14].

\section{SENSOR MANAGER MODULE}

ROBOCAST is a robotic system that assists surgeons in performing brain surgery through a very small hole in the patient's skull (keyhole neurosurgery) for biopsies, Deep Brain Stimulation (DBS) electrodes implantation, and cryogenic and electrolytic ablation.

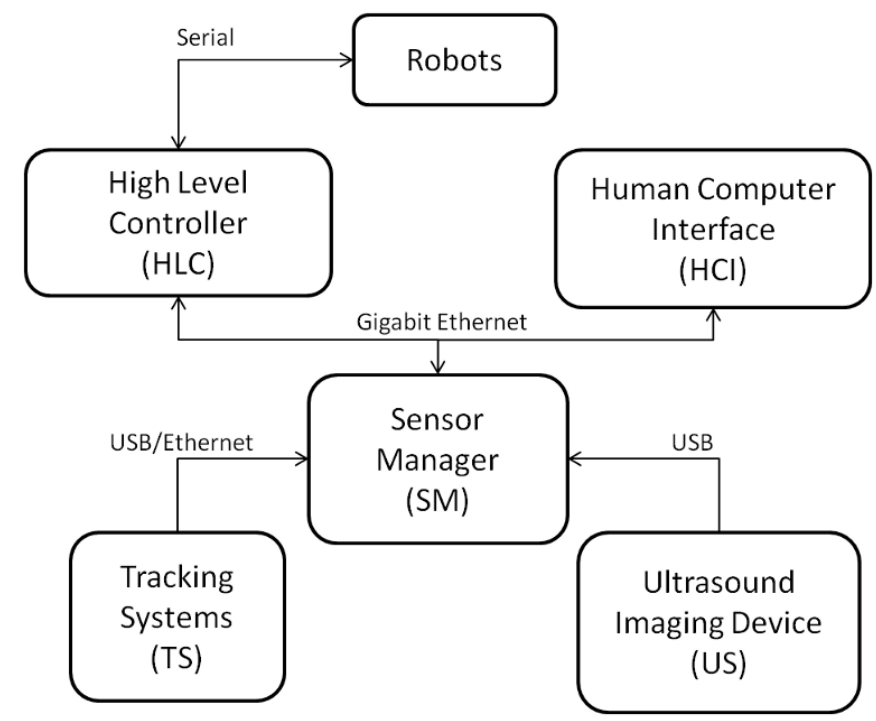

Fig 1. ROBOCAST architecture: TS and US are connected to a laptop where an IGSTK based application (SM) acquires data and forwards it upon request to $\mathrm{HCI}$, a workstation in charge of intra-operative navigation, and to HLC which is responsible for robot control. Communication between SM, $\mathrm{HCI}$, and HCL is achieved through the ACE-TAO CORBA middleware on a Gigabit Ethernet LAN.

The Sensor Manager (SM) is a software component within the distributed architecture of the ROBOCAST system (Fig 1). It is implemented as a $\mathrm{C}++$ service application without a user interface running on a dedicated laptop.
The main purpose of the SM is to gather data from localization systems (optical and electromagnetic) and ultrasound imaging devices, and forward such information upon request to the other components of the system (clients).

As is each component of the ROBOCAST framework, the $\mathrm{SM}$ is part of a client/server architecture based on the middleware TAO CORBA.

The ACE ORB (TAO - The Ace Orb) ${ }^{1}$ is a freelyavailable, open-source, and standards-compliant, real-time $\mathrm{C}++$ implementation of CORBA based upon the Adaptive Communication Environment (ACE). CORBA enables separate pieces of software written in different languages and running on different computers to work with each other like a single application or set of services. More specifically, CORBA is a mechanism in software for normalizing the method-call semantics between application objects residing either in the same address space (application) or remote address space (same host, or remote host on a network). CORBA uses an Interface Definition Language (IDL) to specify the objects' interfaces to the outer world. CORBA then specifies a mapping from IDL to a specific implementation language, such as $\mathrm{C}++$ or Java.

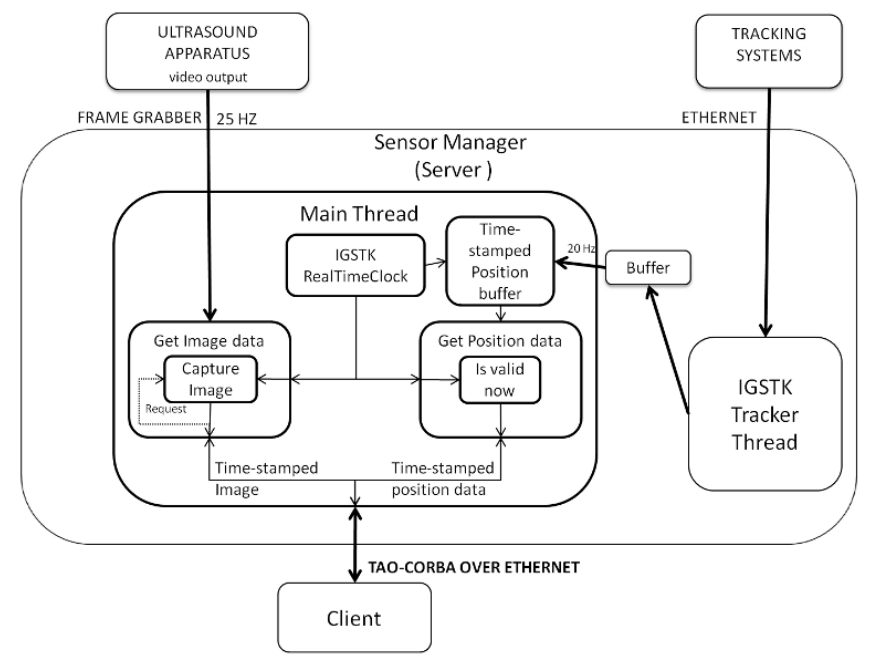

Fig 2. The SM is interfaced with tracking systems through the IGSTK library and with a US machine through the OpenCV library. Client/Server communication is based on the TAO-CORBA Middleware.

The SM encompasses two main parts: (1) interfacing the hardware based on the open source libraries "Image-Guided Surgery ToolKit" (IGSTK) ${ }^{2}$ and OpenCV ${ }^{3}$ and (2) acting as a server to provide data and services to other modules in a CORBA-based network architecture. IGSTK is mainly used for tracking system management and spatial relationship hierarchy among reference frames.

Tracking systems are managed in a transparent way for the user through an $\mathrm{xml}$ configuration file, which allows flexibility in hardware arrangement. IGSTK natively provides support for NDI Aurora, NDI Polaris (Vicra and Spectra), Ascension MedSafe, Ascension trakSTAR, and

\footnotetext{
${ }^{1} \mathrm{http}: / / \mathrm{www} \cdot$ theaceorb.com/

${ }^{2}$ http://www.igstk.org/

${ }^{3} \mathrm{http}: / /$ opencv.willowgarage.com/
} 
Atracsys InfiniTrack among the others; for NDI Optotrak Certus systems, a dedicated class was implemented within the IGSTK framework [14].

According to the IGSTK architecture, as shown in Fig. 2, tracking data is continuously acquired in a separate thread (Tracker Thread, one for each active tracking systems) and stored in a buffer. The main thread updates spatial object transformation, reads the stored tracking data from the buffer at a user-defined frequency $(20 \mathrm{~Hz})$, and marks each data with a timestamp provided by the IGSTK RealTimeClock.

Open-CV allows the acquisition of images from the US device connected to the SM laptop via an USB frame grabber (EZ-Grabber, PAL: 720 x $576 @ 25$ fps). A timestamp is also provided for US image reading time from the unique IGSTK RealTimeClock object instantiated for the whole application.

IGSTK also provides software components to implement a spatial object hierarchy, which facilitates spatial transformation computation between couples of reference frames. This allows the clients to ask for the transformation matrix for a reference system associated with an object in the ROBOCAST scenario, with respect to whatever other object. This is relevant in a multi-sensor system for surgical applications, such as ROBOCAST, where multiple reference frames are involved: optical, electromagnetic, preoperative images (typically T1 MRI and/or CT scans), US images, and actuators' reference frames, which all need to be calibrated to a common reference frame in order to have consistent information. In the ROBOCAST project, the optical reference frame has been chosen as the global reference; calibration matrices have been determined to transform data from any other reference frame to the optical one.

\section{SENSOR MANAGER PERFORMANCES}

The proposed architecture was tested in order to (1) measure the response time needed for the client to receive tracking data or video images and (2) to assess the time lag between synchronous acquisition with an optical tracker and ultrasound machine.

For the first experiment, the setup encompasses an optical localization system (NDI Optotrak Certus), an electromagnetic tracking system (NDI Aurora), an Ultrasound imaging device (Aloka Prosound Alpha 7), the server; a dual-core PC with Windows XP Professional Service Pack 3 (CPU: Intel T7600, 2.33GHz, RAM: 2 GB) running the SM; and a quad-core $\mathrm{PC}$ with Windows XP Professional Service Pack 3 (CPU: Intel Q9550, 2.83GHz, RAM: $2.5 \mathrm{~GB}$ ) running a testing client application. Client and server are connected through the ROBOCAST Gigabit Ethernet LAN based on the TAO-CORBA middleware.

The experimental protocol is the following:

1. On the client, the current time in milliseconds is stored as t1 through the windows API function, which retrieves the current value of the high-resolution performance counter of the personal computer.
2. The client sends a data request to the server with a blocking remote procedure call (RPC) through the TAO-CORBA middleware.

3. The server elaborates the request and sends back data.

4. Once the client receives data, the time ( $\mathrm{t} 2)$ is stored on the client.

5. The time difference $(\mathrm{t} 2-\mathrm{t} 1)$ represents the response time that is composed by request transmission time, server elaboration time, and data transmission time.

Each testing session consists of 30 sets of 200 requests. The experiment was repeated with different tracking systems (NDI Optotrak Certus, NDI Polaris Vicra, and NDI Auora) and with the Aloka Prosound Alpha 7 ultrasound machine.

A Kruskal Wallis test showed no statistical difference between the 30 sets of each system, thus the median value of 6000 samples was computed for each tracking system and for the US machine; results are reported in Table 1.

Table 1. Response time results. Different tracking systems show similar values of response delay; image transmission entails one order of magnitude higher response time.

\begin{tabular}{lc}
\hline Tested system & Response time \\
& (median value and interquartile range) \\
NDI Certus & $1.92 \mathrm{~ms}(1.91-1.94 \mathrm{~ms})$ \\
NDI Aurora & $1.93 \mathrm{~ms}(1.92-1.96 \mathrm{~ms})$ \\
NDI Vicra & $1.94 \mathrm{~ms}(1.91-1.95 \mathrm{~ms})$ \\
Aloka US & $39.86 \mathrm{~ms}(35.37-44.54 \mathrm{~ms})$ \\
\hline
\end{tabular}

In the second experiment, we tested the synchronization of tracking data and US images acquired through the SM module with the following protocol. Again, the hardware adopted consists of the NDI Optotrak Certus optical localization system, the Aloka Prosound Alpha 7 ultrasound imaging device, and the Prosurgics PathFinder serial robot.

The probe, localized with the optical tracker, was immersed in a water tank and moved along the vertical direction using the Pathfinder robot, which was programmed to achieve 3 repetitions of vertical motion (lasting $0.83 \mathrm{~s}$ at $30 \mathrm{~mm} / \mathrm{s}$ constant velocity); the experiment was repeated ten times.

The bottom of the tank is represented on the US image as a line. A mono-dimensional signal was extracted from the image sequence, segmenting the line and calculating the distance in pixels of the central line point from the top of the image. 3D position data of the probe was acquired at the same time and normalized with respect to the amplitude of the performed movement.

The two signals were aligned using the timestamps provided by the SM module, resampled at $1 \mathrm{kHz}$. The time lag was estimated by computing the maximum of the cross correlation function of the two time series. 

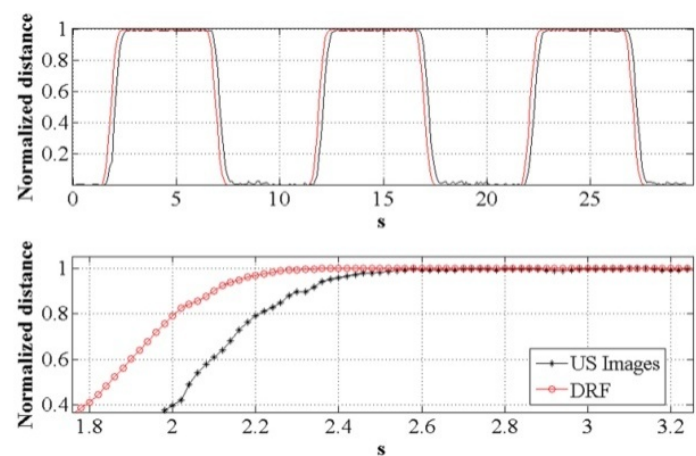

Fig. 3. Monodimensional signals derived from tracking data and US images are reported for a session of three movements (above) and a zoom of the first rising edge (below).

As shown in Fig. 3, we observed a median delay of 230 $\mathrm{ms}$ (first quartile $=223 \mathrm{~ms}$ and third quartile $=240 \mathrm{~ms}$ ).

\section{CONCLUSION}

Navigation in surgery relies on different typologies of sensors, proved to increase the outcome for a huge variety of surgical interventions.

The sensor management architecture we proposed allows for the development of a hardware-independent navigation platform thanks to the transparency of the IGSTK library and of the CORBA middleware. Additionally, the use of open-source software allows the development of a crossplatform, modular application that benefits from a worldwide community of users and developers.

The client/server architecture we proposed showed a transmission delay (between data request and data retrieval on a Gigabit Ethernet network) of approximately $1.9 \mathrm{~ms}$ for the three tested localization systems, which means that a client can request data with a rate up to $500 \mathrm{~Hz}$, much higher than localization systems' frame rate (typically $20-30 \mathrm{~Hz}$ ). Also, US images transmission delay (about $40 \mathrm{~ms}$ ) is in the range of a PAL frame grabber (25 fps).

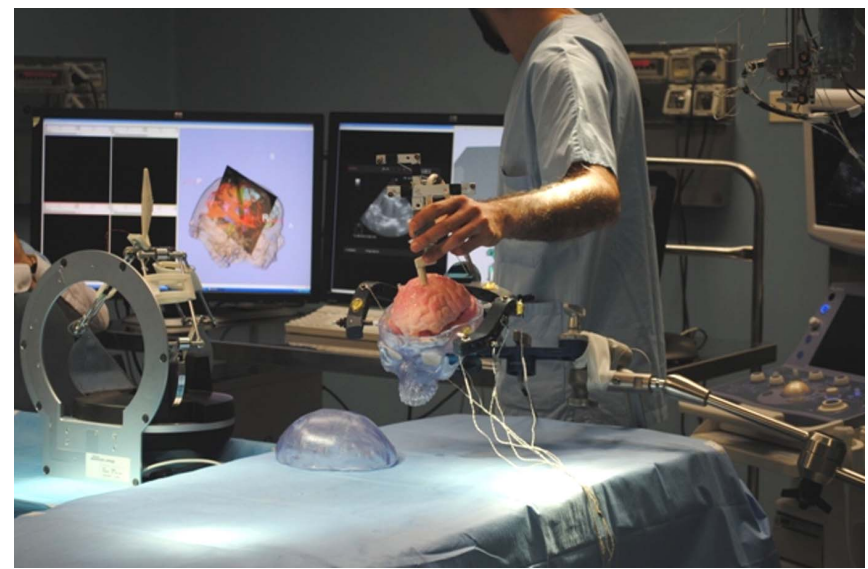

Fig. 4. Intra-operative ultrasound navigation during the ROBOCAST final demo at Ospedale Maggiore, Verona - Italy. Optical tracking of the US probe allows for visualization of the image plane in the 3D scene.

Synchronization between tracking data and US images is assured by the SM module, which associates position data and images with timestamps (only $17 \mathrm{~ms}$ of inter-quartile range shows the delay is approximately constant). A median delay of $230 \mathrm{~ms}$ shows that such architecture does not allow real-time volume reconstruction at this stage, since post processing of data is required for proper time alignment.

Nevertheless, as shown in Fig. 4, a slow exploration (few $\mathrm{mm}$ per second) is indeed possible and useful whenever soft tissue interaction is involved; for instance, during neurosurgical procedures to update the pre-operative plan once brain-shift has occurred. In particular, during asleep patient neurosurgery, brain shift is the main source of inaccuracy, but it occurs with a slow time constant (tens of minutes); in such a scenario, the proposed setup is indeed useful for registration error correction.

\section{REFERENCES}

[1] D. Kendoff, M. Citak, T. Hüfner, S. Chaudhary and C. Krettek, "Current concepts and applications of computer navigation in orthopedic trauma surgery," Central European Journal of Medicine, vol. 2, pp. 392-403, 2007.

[2] E.J. Hanly and M.A. Talamini, "Robotic abdominal surgery," The American Journal of Surgery, vol. 188, pp. 19-26, 2004.

[3] T. Haidegger, L. Kovacs, G. Fordos, Z. Benyo and P. Kazanzides, "Future Trends in Robotic Neurosurgery," in 14th Nordic-Baltic Conference on Biomedical Engineering and Medical Physics, pp. 229233, 2008.

[4] P. Grunert, K. Darabi, J. Espinosa and R. Filippi, "Computer-aided navigation in neurosurgery," Neurosurg. Rev., vol. 26, pp. 73-99, 2003 .

[5] K. Cleary and C. Nguyen, "State of the art in surgical robotics: clinical applications and technology challenges," Computer Aided Surgery, vol. 6, pp. 312-328, 2001.

[6] A. Tobergte, M. Pomarlan and G. Hirzinger, "Robust multi sensor pose estimation for medical applications," in IEEE/RSJ International Conference on Intelligent Robots and Systems, 2009. IROS 2009, pp. 492-497, 2009.

[7] D. De Lorenzo et al. "Miniaturized rigid probe driver with haptic loop control for neurosurgical interventions," in $20103 r d$ IEEE RAS and EMBS International Conference on Biomedical Robotics and Biomechatronics (BioRob), pp. 522-527, 2010.

[8] P.J. Berkelman, L.L. Whitcomb, R.H. Taylor and P. Jensen, "A miniature microsurgical instrument tip force sensor for enhanced force feedback during robot-assisted manipulation," IEEE Trans. on Robotics and Automation, vol. 19, pp. 917-921, 2003.

[9] J. Peirs, J. Clijnen, D. Reynaerts, H.V. Brussel, P. Herijgers, B. Corteville and S. Boone, "A micro optical force sensor for force feedback during minimally invasive robotic surgery," Sensors and Actuators A: Physical, vol. 115, pp. 447-455, 2004.

[10] O. Clatz, H. Delingette, I.F. Talos, A.J. Golby, R. Kikinis, F.A. Jolesz, N. Ayache and S.K. Warfield, "Robust nonrigid registration to capture brain shift from intraoperative MRI," IEEE Transactions on Medical Imaging, vol. 24, pp. 1417-1427, 2005.

[11] R. Bucholz et al., "The correction of stereotactic inaccuracy caused by brain shift using an intraoperative ultrasound device," in CVRMedMRCAS'97, pp. 459-466, 1997.

[12] G. Unsgård, O. Solheim, F. Lindseth and T. Selbekk, "Intra-operative Imaging with 3D Ultrasound in Neurosurgery," Intraoperative Imaging, pp. 181-186, 2011.

[13] M.C. Jacobs and M.A. Livingston, "Managing latency in complex augmented reality systems," in Proceedings of the 1997 symposium on Interactive 3D graphics, pp. 49-ff., 1997.

[14] A. Vaccarella, P. Cerveri, E. De Momi and G. Ferrigno, "A new IGSTK-based architecture for the integration of multimodal sensors and robots in neurosurgical robotics applications," IJCARS, vol. 5, Supplement 1, pp. 308-309, June 23-26. 2010. 